OCHA-PP-133

KEK-TH-614

ICRR-Report-451-99-9

\title{
CP asymmetry for radiative $B$-meson decay in the supersymmetric standard model
}

\author{
Mayumi Aoki用, Gi-Chol Cho ${ }^{2 *}$, and Noriyuki Oshimo ${ }^{3}$ \\ ${ }^{1}$ Graduate School of Humanities and Sciences, Ochanomizu University \\ Bunkyo-ku, Tokyo 112-8610, Japan \\ ${ }^{2}$ Theory Group, KEK, Tsukuba, Ibaraki 305-0801, Japan \\ ${ }^{3}$ Institute for Cosmic Ray Research, University of Tokyo \\ Tanashi, Tokyo 188-8502, Japan
}

\begin{abstract}
We study a rate asymmetry in the inclusive decay $B \rightarrow X_{s} \gamma$, assuming the supersymmetric standard model based on $N=1$ supergravity. A complex coefficient $A$ for scalar-trilinear couplings is the source of $\mathrm{CP}$ violation, which is contained in the mass-squared matrices for squarks. The model parameters are nontrivially constrained by experimental results for the branching ratio of the radiative decay and for the electric dipole moments of the neutron and the electron. The decay rate asymmetry is predicted to be much larger than that by the standard model in a wide region of the parameter space compatible with the experiments. Its magnitude can be maximally around 0.1 , which will be well accessible at B factories.
\end{abstract}

PACS: 11.30.Er, 12.60.Jv, 13.25.Hw, 13.40.Hq

Keywords: $B$ meson, Radiative decay, CP violation, Supersymmetry

* Research Fellow of the Japan Society for the Promotion of Science. 


\section{Introduction}

It has been considered that $\mathrm{CP}$ violation in the $B$-meson system may give an important clue to physics beyond the standard model (SM) [1]. At B factories such $\mathrm{CP}$ asymmetries will be measured in various reactions. One possible candidate is the process $B_{d} \rightarrow \psi K_{S}$, which will be observed with high statistics. In this process the $b$-quark decay occurs dominantly at the tree level by the SM interaction, and new physics could give contributions through $B^{0}-\overline{B^{0}}$ mixing. Another possibility is in the inclusive decay $B \rightarrow X_{s} \gamma$, where the $b$ quark decays at the one-loop level. Although its statistics are not high, new physics could affect the $b$-quark decay.

In this paper, we study a decay rate asymmetry in $B \rightarrow X_{s} \gamma$ within the framework of the supersymmetric standard model (SSM) based on $N=1$ supergravity and grand unified theories (GUTs) [2]. This CP asymmetry is defined as the difference of the decay rates between $\bar{B} \rightarrow X_{s} \gamma$ and its CP conjugate process:

$$
\mathcal{A}_{C P}=\frac{\Gamma\left(\bar{B} \rightarrow X_{s} \gamma\right)-\Gamma\left(B \rightarrow X_{\bar{s}} \gamma\right)}{\Gamma\left(\bar{B} \rightarrow X_{s} \gamma\right)+\Gamma\left(B \rightarrow X_{\bar{s}} \gamma\right)} .
$$

The SM prediction is less than 0.01 [3]. The SSM contains new interactions for the $b$ quark which could sizably induce processes of flavor-changing neutral current (FCNC) [4 5] and of CP violation [6]. We can expect an enhancement of the CP asymmetry for $B \rightarrow X_{s} \gamma$, which indeed has been shown recently [7]. On the other hand, the new interactions for the quarks affect the branching ratio of the radiative decay and the electric dipole moments (EDMs) of the neutron and the electron, both of which have been measured in experiments rather precisely. Taking into account these experimental constraints, we discuss the asymmetry and analyze its dependencies on various SSM parameters. It will be shown that the asymmetry has a magnitude larger than the SM prediction in a wide region of the parameter space allowed by the experiments. Supersymmetry may be revealed through the decay rate asymmetry for $B \rightarrow X_{s} \gamma$ at $\mathrm{B}$ factories.

The decay $B \rightarrow X_{s} \gamma$ is well described by the quark-level processes $b \rightarrow s \gamma$ and $b \rightarrow s \gamma g$, owing to a large mass of the $b$ quark. In the SSM, there are several new sources of FCNC which give rise to these decays at the one-loop level. In particular, the interactions of down-type quarks with charginos and up-type squarks and with charged Higgs bosons and up-type quarks play dominant roles [4]. Furthermore, the former interactions violate CP invariance [6], which is attributed to the chargino mass matrix or the $t$-squark mass-squared matrix. We study the effects of these interactions on the $\mathrm{CP}$ asymmetry. Although the interactions of 
down-type quarks with gluinos and down-type squarks or with neutralinos and down-type squarks could also become sources of FCNC and CP violation, their effects are suppressed by small off-diagonal elements of the $b$-squark mass-squared matrix.

Experiments have put stringent bounds on the branching ratio of $B \rightarrow X_{s} \gamma$ and the EDMs of the neutron and the electron. For the branching ratio, two measurements have been reported by CLEO [8] and ALEPH [9] to give respectively

$$
\begin{aligned}
\operatorname{Br}\left(B \rightarrow X_{s} \gamma\right) & =(3.15 \pm 0.35 \pm 0.32 \pm 0.26) \times 10^{-4} \\
& =(3.11 \pm 0.80 \pm 0.72) \times 10^{-4}
\end{aligned}
$$

Both results are consistent with the prediction of the SM including next-to-leading order $(\mathrm{NLO})$ corrections for $\mathrm{QCD}, \operatorname{Br}\left(B \rightarrow X_{s} \gamma\right)=(3.29 \pm 0.33) \times 10^{-4}$ [10]. The experimental upper bounds on the neutron and the electron EDMs have been obtained as $\left|d_{n}\right| \lesssim 10^{-25} e \mathrm{~cm}$ [1] and $\left|d_{e}\right| \lesssim 10^{-26} e \mathrm{~cm}$ [12]. The SM predictions are

given by $\left|d_{n}\right|=10^{-33}-10^{-31} e \mathrm{~cm}$ [13] and $\left|d_{e}\right| \lesssim 10^{-37} e \mathrm{~cm}$ [14]. These experimental results lead to useful constraints on SSM parameters.

This paper is organized as follows. In Section 2, we summarize sources of CP violation and FCNC in the SSM and present the interaction Lagrangians which are relevant to the decay $B \rightarrow X_{s} \gamma$. In Section 3, Wilson coefficients for the radiative decay are obtained and formulae for the CP asymmetry are given explicitly in a self-contained form. In Section 4, numerical results for the CP asymmetry are presented together with the branching ratio. Discussions and conclusions are given in Section 5.

\section{Sources of CP violation and FCNC}

In the SSM coupled to $N=1$ supergravity, there are many complex parameters, as well as Yukawa coupling constants: a mass parameter $m_{H}$ in the bilinear term of the Higgs superfields, $\mathrm{SU}(3), \mathrm{SU}(2)$, and $\mathrm{U}(1)$ gaugino masses $\widetilde{m}_{3}, \widetilde{m}_{2}$, and $\widetilde{m}_{1}$, and dimensionless coefficients $A_{f}$ and $B$ for scalar-trilinear and -bilinear couplings, $f$ representing a flavor for quarks and leptons. Assuming that the gaugino masses have a common value at around the GUT scale, we take

$$
\frac{3 \widetilde{m}_{1}}{5 \alpha_{1}}=\frac{\widetilde{m}_{2}}{\alpha_{2}}=\frac{\widetilde{m}_{3}}{\alpha_{3}}
$$


at the electroweak scale. We also assume that the differences of $A_{f}$ among flavors are small, putting the same value on them $A_{f}=A$. Then only two new complex phases become physical. By appropriate field redefinitions, we can take without loss of generality $m_{H}$ and $A$ as complex,

$$
m_{H}=\left|m_{H}\right| \exp (i \theta), \quad A=|A| \exp (i \alpha)
$$

and the gaugino masses and $B m_{H}$ as real. The vacuum expectation values $v_{1}$ and $v_{2}$ of the Higgs bosons with hypercharges $Y=-1 / 2$ and $+1 / 2$, respectively, become real in this convention.

The new source of FCNC relevant to $B \rightarrow X_{s} \gamma$ is the mass-squared matrix $M_{U}^{2}$ for up-type squarks, which contains the physical complex phases $\alpha$ and $\theta$ intrinsic in the SSM and thus also becomes an origin of CP violation. Since the squarks are mixed not only among different generations but also between left- and right-handed components, $M_{U}^{2}$ is expressed by a $(6 \times 6)$ matrix. We assume that the generation mixings in $M_{U}^{2}$ can be removed approximately by the same unitary matrices which diagonalize the mass matrix of up-type quarks. Then, the generation mismatches between up-type squarks and down-type quarks in the interactions with charginos are described by the Cabibbo-Kobayashi-Maskawa (CKM) matrix for the quarks.

The magnitude of the left-right mixing for the squarks $\widetilde{q}_{k}$ is proportional to the Yukawa coupling constant for the corresponding quark $q$. Consequently, in the first two generations, the left-right mixings are safely neglected. The lefthanded squarks $\widetilde{u}_{L}, \widetilde{c}_{L}$ and the right-handed squarks $\widetilde{u}_{R}, \widetilde{c}_{R}$ are themselves in mass eigenstates, whose masses are given by

$$
\begin{aligned}
& \widetilde{M}_{u L}^{2}=\widetilde{M}_{c L}^{2}=\widetilde{m}_{Q}^{2}+\cos 2 \beta\left(\frac{1}{2}-\frac{2}{3} \sin ^{2} \theta_{W}\right) M_{Z}^{2} \\
& \widetilde{M}_{u R}^{2}=\widetilde{M}_{c R}^{2}=\widetilde{m}_{U}^{2}+\frac{2}{3} \cos 2 \beta \sin ^{2} \theta_{W} M_{Z}^{2} .
\end{aligned}
$$

Here $\widetilde{m}_{Q}$ and $\widetilde{m}_{U}$ denote soft supersymmetry-breaking masses for the squarks of $\mathrm{SU}(2)$ doublets and singlets, respectively, and have roughly the same value. The angle $\beta$ is defined by $\tan \beta=v_{2} / v_{1}$. The $u$ - and $c$-quark masses have been neglected.

For the squarks of the third generation $\widetilde{t}_{L}$ and $\widetilde{t}_{R}$, the left-right mixing is no longer negligible, because of a large Yukawa coupling constant for the $t$ quark . The mass-squared matrix $M_{t}^{2}$ for the $t$ squarks is given by

$$
M_{t}^{2}=\left(\begin{array}{cc}
\widetilde{M}_{u L}^{2}+(1-c) m_{t}^{2} & m_{t}\left(A^{*} m_{3 / 2}+\cot \beta m_{H}\right) \\
m_{t}\left(A m_{3 / 2}+\cot \beta m_{H}^{*}\right) & \widetilde{M}_{u R}^{2}+(1-2 c) m_{t}^{2}
\end{array}\right),
$$


where $m_{3 / 2}$ represents the gravitino mass, satisfying $\widetilde{m}_{Q} \approx \widetilde{m}_{U} \approx|A| m_{3 / 2}$. A dimensionless constant $c$ is introduced to parametrize radiative corrections to the squark masses through Yukawa interactions, with $c=0.1-1$. The mass eigenstates of the $t$ squarks $\widetilde{t}_{1}$ and $\widetilde{t}_{2}$ are obtained by diagonalizing $M_{t}^{2}$ as

$$
S_{t}^{\dagger} M_{t}^{2} S_{t}=\operatorname{diag}\left(\widetilde{M}_{t 1}^{2}, \widetilde{M}_{t 2}^{2}\right) \quad\left(\widetilde{M}_{t 1}^{2}<\widetilde{M}_{t 2}^{2}\right)
$$

where $S_{t}$ is a unitary matrix.

The CP-violating phase $\theta$ also appears in the mass matrix for charginos $\omega_{i}$, which are charged mass eigenstates of $\mathrm{SU}(2)$ gauginos and Higgsinos. The mass matrix is given by

$$
M^{-}=\left(\begin{array}{cc}
\widetilde{m}_{2} & -\sqrt{2} \cos \beta M_{W} \\
-\sqrt{2} \sin \beta M_{W} & m_{H}
\end{array}\right) .
$$

The mass eigenstates are obtained by diagonalizing $M^{-}$as

$$
C_{R}^{\dagger} M^{-} C_{L}=\operatorname{diag}\left(m_{\omega 1}, m_{\omega 2}\right) \quad\left(m_{\omega 1}<m_{\omega 2}\right)
$$

where $C_{R}$ and $C_{L}$ are unitary matrices.

The interaction Lagrangians which could give large new contributions to the decays $b \rightarrow s \gamma$ and $b \rightarrow s \gamma g$ are given as follows [4, 5]:

The chargino - quark - squark interactions

$$
\begin{aligned}
\mathcal{L} & =i \frac{g}{\sqrt{2}} \sum_{i=1}^{2}\left\{\left(\widetilde{u}_{L}^{\dagger}, \widetilde{c}_{L}^{\dagger}, \sum_{k=1}^{2} S_{t 1 k}^{*} \widetilde{t}_{k}^{\dagger}\right) V \bar{\omega}_{i}\left(\sqrt{2} C_{R 1 i}^{*} \frac{1-\gamma_{5}}{2}+\frac{\bar{m}_{D}}{M_{W}} \frac{C_{L 2 i}^{*}}{\cos \beta} \frac{1+\gamma_{5}}{2}\right)\left(\begin{array}{c}
d \\
s \\
b
\end{array}\right)\right. \\
& \left.-\left(\widetilde{u}_{R}^{\dagger}, \widetilde{c}_{R}^{\dagger}, \sum_{k=1}^{2} S_{t 2 k}^{*} \widetilde{t}_{k}^{\dagger}\right) \frac{C_{R 2 i}^{*}}{\sin \beta} \frac{\bar{m}_{U}}{M_{W}} V \bar{\omega}_{i} \frac{1-\gamma_{5}}{2}\left(\begin{array}{c}
d \\
s \\
b
\end{array}\right)\right\}+ \text { h.c. },
\end{aligned}
$$

The charged Higgs boson - quark - quark interactions

$$
\begin{aligned}
\mathcal{L}= & \frac{g}{\sqrt{2}} H^{+}(\bar{u}, \quad \bar{c}, \bar{t})\left(\cot \beta \frac{\bar{m}_{U}}{M_{W}} V \frac{1-\gamma_{5}}{2}+\tan \beta V \frac{\bar{m}_{D}}{M_{W}} \frac{1+\gamma_{5}}{2}\right)\left(\begin{array}{c}
d \\
s \\
b
\end{array}\right) \\
& + \text { h.c.. }
\end{aligned}
$$

Here $V$ stands for the CKM matrix and $\bar{m}_{U}$ and $\bar{m}_{D}$ represent the diagonalized quark mass matrices

$$
\bar{m}_{U}=\operatorname{diag}\left(0,0, m_{t}\right), \quad \bar{m}_{D}=\operatorname{diag}\left(0,0, m_{b}\right),
$$


with the quark masses of the first two generations being neglected.

Rigorous constraints on the new CP-violating phases $\theta$ and $\alpha$ come from the EDMs of the neutron and the electron. The EDMs receive contributions from one-loop diagrams mediated by squarks or sleptons with gluinos, charginos, or neutralinos. If $\mathrm{CP}$ violation by the phase $\theta$ is not suppressed, the experimental bounds on the EDMs constrain squark and slepton masses to be larger than 1 $\mathrm{TeV}$ [15]. On the other hand, the phase $\alpha$ is allowed to maximally cause CP violation even if squarks and sleptons are not heavy [6, 16]. Only the gluinos are required to be sufficiently heavy, leading to $\widetilde{m}_{2} \gtrsim 500 \mathrm{GeV}$ from Eq. (2.1). The decay rate asymmetry in Eq. (1.1) is expected to have a large magnitude only if at least one CP-violating phase is not suppressed and the charginos and the squarks are not heavy. We therefore assume that $\theta$ is about 0 or $\pi$, while no constraint is imposed on $\alpha$.

\section{Decay rate asymmetry and branching ratio}

The radiative decay $B \rightarrow X_{s} \gamma$ can be approximated by the free quark processes $b \rightarrow s \gamma$ and $b \rightarrow s \gamma g$. These elementary processes for the $\Delta B=1$ transition are studied by using the effective Hamiltonian with five quarks in which heavier degrees of freedom are integrated out:

$$
\mathcal{H}_{e f f}=-\frac{4 G_{F}}{\sqrt{2}} V_{t s}^{*} V_{t b} \sum_{j=1}^{8} C_{j}(\mu) O_{j}(\mu) .
$$

Here $O_{j}(\mu)$ and $C_{j}(\mu)$ represent an operator for the $\Delta B=1$ transition and its Wilson coefficient, respectively, evaluated at an energy scale $\mu$. The relevant operators for $B \rightarrow X_{s} \gamma$ are given by

$$
\begin{aligned}
O_{2} & =\overline{s_{L}} \gamma^{\mu} c_{L} \overline{c_{L}} \gamma_{\mu} b_{L}, \\
O_{7} & =\frac{e}{16 \pi^{2}} m_{b} \overline{s_{L}} \sigma^{\mu \nu} b_{R} F_{\mu \nu}, \\
O_{8} & =\frac{g_{s}}{16 \pi^{2}} m_{b} \overline{s_{L}} \sigma^{\mu \nu} T^{\alpha} b_{R} G_{\mu \nu}^{\alpha},
\end{aligned}
$$

where $F_{\mu \nu}$ and $G_{\mu \nu}^{\alpha}$ respectively represent the electromagnetic and the strong field strength tensors, $T^{\alpha}$ being a generator for $\mathrm{SU}(3)$. The CP asymmetry and branching ratio for $B \rightarrow X_{s} \gamma$ are expressed in terms of the Wilson coefficients at $\mu=m_{b}$.

In matching the SSM to the effective Hamiltonian at $\mu=M_{W}$, the coefficients $C_{7}$ and $C_{8}$ receive contributions from one-loop diagrams which are mediated by 
charginos and charged Higgs bosons with up-type squarks and up-type quarks, respectively, as well as by $W$ bosons with up-type quarks. The relevant Feynman diagrams are shown in Fig. 1. The coefficients $C_{2}\left(M_{W}\right), C_{7}\left(M_{W}\right)$, and $C_{8}\left(M_{W}\right)$ are then expressed at the leading order $(\mathrm{LO})$ as

$$
\begin{aligned}
& C_{2}\left(M_{W}\right)=1, \\
& C_{7}\left(M_{W}\right)=C_{7}^{W}\left(M_{W}\right)+C_{7}^{H^{ \pm}}\left(M_{W}\right)+C_{7}^{\omega}\left(M_{W}\right), \\
& C_{8}\left(M_{W}\right)=C_{8}^{W}\left(M_{W}\right)+C_{8}^{H^{ \pm}}\left(M_{W}\right)+C_{8}^{\omega}\left(M_{W}\right),
\end{aligned}
$$

where $C_{j}^{W}\left(M_{W}\right), C_{j}^{H^{ \pm}}\left(M_{W}\right)$, and $C_{j}^{\omega}\left(M_{W}\right)(j=7,8)$ denote, respectively, the contributions from $W$ bosons, charged Higgs bosons, and charginos. From the interaction Lagrangian in Eq. (2.8), we obtain the chargino contributions as

$$
\begin{aligned}
C_{j}^{\omega}\left(M_{W}\right)= & \sum_{i=1}^{2} \frac{M_{W}^{2}}{m_{\omega i}^{2}}\left[-\left|C_{R 1 i}\right|^{2} r_{u i} K_{1}^{j}\left(r_{u i}\right)-\frac{C_{R 1 i} C_{L 2 i}^{*}}{\sqrt{2} \cos \beta} \frac{m_{\omega i}}{M_{W}} r_{u i} K_{2}^{j}\left(r_{u i}\right)\right. \\
& +\sum_{k=1}^{2}\left\{\left|C_{R 1 i} S_{t 1 k}-\frac{C_{R 2 i} S_{t 2 k}}{\sqrt{2} \sin \beta} \frac{m_{t}}{M_{W}}\right|^{2} r_{k i} K_{1}^{j}\left(r_{k i}\right)\right. \\
& \left.\left.+\frac{C_{L 2 i}^{*} S_{t 1 k}^{*}}{\sqrt{2} \cos \beta}\left(C_{R 1 i} S_{t 1 k}-\frac{C_{R 2 i} S_{t 2 k}}{\sqrt{2} \sin \beta} \frac{m_{t}}{M_{W}}\right) \frac{m_{\omega i}}{M_{W}} r_{k i} K_{2}^{j}\left(r_{k i}\right)\right\}\right], \\
r_{u i}= & \frac{m_{\omega i}^{2}}{\widetilde{M}_{u L}^{2}}, \quad r_{k i}=\frac{m_{\omega i}^{2}}{\widetilde{M}_{t k}^{2}}, \\
K_{a}^{7}(r)= & I_{a}(r)+\frac{2}{3} J_{a}(r), \quad K_{a}^{8}(r)=J_{a}(r), \quad(a=1,2)
\end{aligned}
$$

where the functions $I_{a}(r)$ and $J_{a}(r)$ are defined by

$$
\begin{aligned}
& I_{1}(r)=\frac{1}{12(1-r)^{4}}\left(2+3 r-6 r^{2}+r^{3}+6 r \ln r\right), \\
& I_{2}(r)=\frac{1}{2(1-r)^{3}}\left(-3+4 r-r^{2}-2 \ln r\right), \\
& J_{1}(r)=\frac{1}{12(1-r)^{4}}\left(1-6 r+3 r^{2}+2 r^{3}-6 r^{2} \ln r\right), \\
& J_{2}(r)=\frac{1}{2(1-r)^{3}}\left(1-r^{2}+2 r \ln r\right) .
\end{aligned}
$$

The contributions of charged Higgs bosons are given from Eq. (2.9) by

$$
\begin{aligned}
C_{j}^{H^{ \pm}}\left(M_{W}\right) & =-\frac{1}{2} r_{H}\left\{\cot ^{2} \beta \bar{K}_{1}^{j}\left(r_{H}\right)+\bar{K}_{2}^{j}\left(r_{H}\right)\right\}, \\
r_{H} & =\frac{m_{t}^{2}}{M_{H^{ \pm}}^{2}}, \\
\bar{K}_{a}^{7}(r) & =\frac{2}{3} I_{a}(r)+J_{a}(r), \quad \bar{K}_{a}^{8}(r)=I_{a}(r),
\end{aligned}
$$


and the standard $W$-boson contributions lead to

$$
\begin{aligned}
C_{j}^{W}\left(M_{W}\right) & =-\frac{3}{2} r_{W} \bar{K}_{1}^{j}\left(r_{W}\right), \\
r_{W} & =\frac{m_{t}^{2}}{M_{W}^{2}} .
\end{aligned}
$$

The coefficients $C_{j}^{W}\left(M_{W}\right)$ and $C_{j}^{H^{ \pm}}\left(M_{W}\right)$ have real values, whereas $C_{j}^{\omega}\left(M_{W}\right)$ are complex, leading to $\mathrm{CP}$ violation.

An important point is that the chargino contribution can work both constructively and destructively. The charged Higgs-boson contribution has the same sign as the $W$-boson contribution, so that their sum is larger in magnitude than the latter alone, $\left|C_{j}^{W}\left(M_{W}\right)+C_{j}^{H^{ \pm}}\left(M_{W}\right)\right|>\left|C_{j}^{W}\left(M_{W}\right)\right|$. On the other hand, owing to a complex value of $C_{j}^{\omega}\left(M_{W}\right)$, the total sum $C_{j}\left(M_{W}\right)$ may be larger or may be smaller in magnitude than $C_{j}^{W}\left(M_{W}\right)$ depending on the parameter values. It is possible that the conditions $\left|C_{j}^{\omega}\left(M_{W}\right)\right| \sim\left|C_{j}^{W}\left(M_{W}\right)\right|$ and $\left|C_{j}\left(M_{W}\right)\right| \approx\left|C_{j}^{W}\left(M_{W}\right)\right|$ are satisfied simultaneously. In this case $\mathrm{CP}$ invariance is violated maximally. Even if the branching ratio of $B \rightarrow X_{s} \gamma$ is comparable with the SM value, its CP asymmetry may have a large magnitude.

The Wilson coefficients at $\mu=m_{b}$ are obtained by solving the renormalization group equations

$$
\mu \frac{d}{d \mu} C_{i}(\mu)=C_{j}(\mu) \gamma_{j i},
$$

where $\gamma$ is the anomalous dimension matrix. From the LO anomalous dimension matrix the coefficients become

$$
\begin{aligned}
& C_{2}\left(m_{b}\right)=\frac{1}{2}\left(\eta^{-\frac{12}{23}}+\eta^{\frac{6}{23}}\right) \\
& C_{7}\left(m_{b}\right)=\eta^{\frac{16}{23}} C_{7}\left(M_{W}\right)+\frac{8}{3}\left(\eta^{\frac{14}{23}}-\eta^{\frac{16}{23}}\right) C_{8}\left(M_{W}\right)+\sum_{i=1}^{8} h_{i} \eta^{a_{i}}, \\
& C_{8}\left(m_{b}\right)=\eta^{\frac{14}{23}} C_{8}\left(M_{W}\right)+\sum_{i=1}^{8} \bar{h}_{i} \eta^{a_{i}},
\end{aligned}
$$

with $\eta=\alpha_{s}\left(M_{W}\right) / \alpha_{s}\left(m_{b}\right)$ which is set for $\eta=0.56$ in the following numerical study. The constants $h_{i}, \bar{h}_{i}$, and $a_{i}$ are listed in Table 1 [17].

The rate asymmetry in the decay $B \rightarrow X_{s} \gamma$ is attributed to decay rate asymmetries for $b \rightarrow s \gamma$ and $b \rightarrow s \gamma g$, which are induced by the interferences between the tree diagrams and the loop diagrams with absorptive parts as shown in Fig. 2 . 
Combining these two contributions, the asymmetry in Eq. (1.1) is given by [18, 19]

$$
\begin{aligned}
\mathcal{A}_{C P}= & \frac{4 \alpha_{s}\left(m_{b}\right)}{9\left|C_{7}\left(m_{b}\right)\right|^{2}}\left(\left[\frac{10}{9}-2 z\{v(z)+b(z, \delta)\}\right] \operatorname{Im}\left[C_{2}\left(m_{b}\right) C_{7}^{*}\left(m_{b}\right)\right]\right. \\
& \left.+\operatorname{Im}\left[C_{7}\left(m_{b}\right) C_{8}^{*}\left(m_{b}\right)\right]+\frac{2}{3} z b(z, \delta) \operatorname{Im}\left[C_{2}\left(m_{b}\right) C_{8}^{*}\left(m_{b}\right)\right]\right), \\
v(z)= & \left(5+\ln z+\ln ^{2} z-\frac{\pi^{2}}{3}\right)+\left(\ln ^{2} z-\frac{\pi^{2}}{3}\right) z \\
& +\left(\frac{28}{9}-\frac{4}{3} \ln z\right) z^{2}+O\left(z^{3}\right), \\
b(z, \delta)= & g(z, 1)-g(z, 1-\delta), \\
g(z, y)= & \theta(y-4 z)\left\{\left(y^{2}-4 y z+6 z^{2}\right) \ln \left(\sqrt{\frac{y}{4 z}}+\sqrt{\frac{y}{4 z}-1}\right)\right. \\
& \left.\quad-\frac{3 y(y-2 z)}{4} \sqrt{1-\frac{4 z}{y}}\right\},
\end{aligned}
$$

with $z=m_{c}^{2} / m_{b}^{2}$. We have neglected the effect of the standard CP-violating phase in the CKM matrix, which is known to be small [3]. Owing to the contribution of the three-body decay $b \rightarrow s \gamma g$, the emitted photon in the inclusive decay process $B \rightarrow X_{s} \gamma$ has a continuous energy spectrum. The parameter $\delta$ expresses the cut for the photon energy, $E_{\gamma}>(1-\delta) m_{b} / 2$, which is assumed in calculating the asymmetry.

The theoretical prediction of the branching ratio has large perturbative uncertainties at the LO, which are significantly reduced by taking into account NLO corrections. To the present, these corrections have been calculated for the matrix elements at $\mu=m_{b}$ [19, 20] and the anomalous dimensions [21]. For the matching conditions of the Wilson coefficients at $\mu=M_{W}$, calculations have been performed completely in the SM [22]. However, in the SSM, the conditions have only been obtained for limited cases [23, 24], which are not generally applicable. We, therefore, calculate the branching ratio of $B \rightarrow X_{s} \gamma$ by using the matrix elements and the anomalous dimensions with NLO corrections, while for the matching conditions NLO corrections are taken into account only for the $W$-boson contributions. Our calculations follow formulae given in Ref. [10, which include also QED corrections.

\section{Numerical study}

We present numerical results of the decay rate asymmetry for $B \rightarrow X_{s} \gamma$ together with its branching ratio. Taking into account experimental constraints on the 
EDMs of the neutron and the electron, we assume that CP violation is induced solely by the phase $\alpha$ in Eq. (2.2) and the $\mathrm{SU}(2)$ gaugino mass $\widetilde{m}_{2}$ is sufficiently large. In the following analyses, we fix its value as $\widetilde{m}_{2}=500 \mathrm{GeV}$, though the asymmetry and the branching ratio do not depend much on $\widetilde{m}_{2}$. The mass ranges $\widetilde{M}_{t 1}<80 \mathrm{GeV}$ [25] and $m_{\omega 1}<70 \mathrm{GeV}$ [26] are experimentally excluded for the lighter $t$ squark $\tilde{t}_{1}$ and the lighter chargino $\omega_{1}$, respectively, by direct searches for supersymmetry particles. In addition, it is imposed that $\tilde{t}_{1}$ should be heavier than the lightest neutralino from cosmological consideration. For simplicity, we assume that the soft supersymmetry-breaking masses $\widetilde{m}_{Q}$ and $\widetilde{m}_{U}$ have the same value, and define a ratio $R=\widetilde{m}_{Q} /|A| m_{3 / 2}=\widetilde{m}_{U} /|A| m_{3 / 2}$. For definiteness, measurable parameters are fixed at the $b$-quark mass scale as the followings: $m_{b}=4.8 \mathrm{GeV}$, $z=m_{c}^{2} / m_{b}^{2}=(0.29)^{2},\left|V_{t s}^{*} V_{t b} / V_{c b}\right|=0.97$. The energy cutoff parameter $\delta$ for the photon is taken to be 0.99 , though the decay rate asymmetry is not changed so much by the choice of its value.

The decay rate asymmetry and the branching ratio are shown in Fig. 3 as functions of the lighter $t$-squark mass $\widetilde{M}_{t_{1}}$ for (a) $\tan \beta=2$, (b) $\tan \beta=10$, and (c) $\tan \beta=30$. We set the CP-violating phases for $\alpha=\pi / 4$ and $\theta=0$. The mass parameters for the charged Higgs boson and the Higgsino are taken for $M_{H^{ \pm}}=200 \mathrm{GeV}$ and $\left|m_{H}\right|=100 \mathrm{GeV}$. The ratio $R$ is set for $R=1$. The parameters $c$ and $|A| m_{3 / 2}$ are scanned over $0.1 \leq c \leq 1$ and $|A| m_{3 / 2} \leq 1 \mathrm{TeV}$. The experimental upper and lower bounds of the branching ratio 8, 9] are also indicated. For $\tan \beta=10$, the branching ratio lies within the experimental bounds by ALEPH in the range $100 \mathrm{GeV} \lesssim \widetilde{M}_{t 1} \lesssim 400 \mathrm{GeV}$, where the asymmetry has a value $0.02 \lesssim\left|\mathcal{A}_{C P}\right| \lesssim 0.07$. The peaks of the asymmetry and the branching ratio are both roughly given at $\widetilde{M}_{t 1} \simeq 200 \mathrm{GeV}$. For $\tan \beta=2$ and $\tan \beta=30$, the branching ratio is consistent with its experimental bounds in the ranges 100 $\mathrm{GeV} \lesssim \widetilde{M}_{t 1} \lesssim 200 \mathrm{GeV}$ and $300 \mathrm{GeV} \lesssim \widetilde{M}_{t 1} \lesssim 700 \mathrm{GeV}$, where the asymmetry has values $0.01 \lesssim\left|\mathcal{A}_{C P}\right| \lesssim 0.06$ and $0.02 \lesssim\left|\mathcal{A}_{C P}\right| \lesssim 0.075$, respectively. The peaks of the asymmetry and the branching ratio are roughly at the same value of $\widetilde{M}_{t 1}$, which increases with $\tan \beta$. The maximal value of the asymmetry does not depend significantly on $\tan \beta$. As $\tan \beta$ increases, larger masses for $\tilde{t}_{1}$ become allowed by the experimental constraints.

We show in Fig. $⿴$ the asymmetry and the branching ratio for (a) $R=0.5$ and (b) $R=2$, with $\tan \beta=10$. The other parameters are taken for the same values as in Fig. 3. The allowed region for the mass of $\tilde{t}_{1}$ is given by $150 \mathrm{GeV} \lesssim \widetilde{M}_{t 1} \lesssim 500$ 
$\mathrm{GeV}$ for $R=0.5$, where the asymmetry is in the range $0.02 \lesssim\left|\mathcal{A}_{C P}\right| \lesssim 0.075$. For $R=2$, under the condition that $\widetilde{M}_{t 1}$ remain the same, the amount of mixing for $\tilde{t}_{L}$ and $\tilde{t}_{R}$ varies very much with $c$, leading to strong $c$-dependencies of the asymmetry and the branching around their peaks. The asymmetry becomes $0.01 \lesssim\left|\mathcal{A}_{C P}\right| \lesssim 0.065$.

In Fig. 5, the asymmetry and the branching ratio are shown for $M_{H^{ \pm}}=500$ $\mathrm{GeV}$, with $\tan \beta=10$. We take the other parameters for the same values as in Fig. 3. Since the charged Higgs-boson contribution to $b \rightarrow s \gamma$ decreases as $M_{H^{ \pm}}$ becomes larger, the branching ratio is smaller than that for $M_{H^{ \pm}}=200 \mathrm{GeV}$. As a result, a large value of $\widetilde{M}_{t 1}$ becomes allowed by the experimental constraints for the branching ratio, whereas for $150 \mathrm{GeV} \lesssim \widetilde{M}_{t 1} \lesssim 300 \mathrm{GeV}$ the branching ratio is too small. The asymmetry is given by $\left|\mathcal{A}_{C P}\right| \lesssim 0.06$ in the experimentally allowed range, and does not vary much with $M_{H^{ \pm}}$.

We plot in Fig. 6 the asymmetry and the branching ratio as functions of the Higgsino mass parameter $m_{H}$ for (a) $\alpha=\pi / 4$ and (b) $\alpha=3 \pi / 4$, with $\tan \beta=10$. The mass of $\tilde{t}_{1}$ is kept for $195 \mathrm{GeV} \leq \widetilde{M}_{t 1} \leq 205 \mathrm{GeV}$. The phase $\theta$ is taken for 0 or $\pi$, corresponding to positive or negative values for $m_{H}$, respectively. The other parameters are taken for the same values as in Fig. 3. The ranges for smaller values of $\left|m_{H}\right|$, where no plot is given, are excluded by the experiments since $m_{\omega 1}$ becomes less than $70 \mathrm{GeV}$. In the ranges for larger values of $\left|m_{H}\right|$ with no plot, the lightest neutralino is heavier than $\tilde{t}_{1}$. For $\alpha=\pi / 4$, in the range with $m_{H}>0$ the chargino contributions to $C_{7}$ and $C_{8}$ are added destructively to the standard $W$-boson and the charged Higgs-boson contributions. On the other hand, in the range with $m_{H}<0$ the formers are added constructively to the latters, making the branching ratio too large. Such a relative relation depends on not only $\theta$ but also $\alpha$. For $\alpha=3 \pi / 4$, the branching ratio is too large for $m_{H}>0$ while within the experimental bounds for $m_{H}<0$. For either value of $\alpha$, the asymmetry is given by $0.02 \lesssim\left|\mathcal{A}_{C P}\right| \lesssim 0.075$ in the experimentally allowed ranges.

Summarizing these numerical results, the absolute value of the decay rate asymmetry in the SSM is larger than 0.02 in wide parameter regions where the branching ratio is consistent with its experimental bounds. If $M_{H^{ \pm}}$is of order $100 \mathrm{GeV}$, the charged Higgs-boson contribution to $B \rightarrow X_{s} \gamma$ is not small [24]. Since the experimental values for the branching ratio are almost consistent with the SM prediction, the chargino-squark loop diagram has to give a large destructive contribution in order to cancel out the large charged Higgs-boson contribution. Therefore, as long 
as the CP-violating phase $\alpha$ is not suppressed, the chargino contribution induces a sizable CP asymmetry. On the other hand, if the charged Higgs boson is heavy, its contribution becomes negligible. Still, as shown in Fig. 5, there are parameter regions where the asymmetry can be sizable without conflicting with the measured branching ratio. Because it is possible that the chargino contributions make the Wilson coefficients $C_{7}$ and $C_{8}$ complex while their absolute values being kept unchanged.

\section{Discussions}

The decay rate asymmetry for $B \rightarrow X_{s} \gamma$ could be measured at $\mathrm{B}$ factories. It is expected there that $B \bar{B}$ pairs of order $10^{8}$ will be produced per year, among which approximately half are pairs of $B^{+} B^{-}$. Since $B^{0}$ and $\bar{B}^{0}$ may make the transition to each other before their decays, $B^{+} B^{-}$pairs are suitable for the measurement of the asymmetry discussed in this paper. For estimating measurability we tentatively assume that the tagging efficiency of $B \rightarrow X_{s} \gamma$ by detecting the photon is around 0.3. A distinction between $B^{+}$and $B^{-}$can be made by examining the strange particle accompanied with the photon or the semileptonic decay of the opposite $B$ meson. We assume these tagging efficiencies to be around 0.1 each. Then, a detectable number of $B \rightarrow X_{s} \gamma$ with the charge of $B$ being specified is roughly of order $10^{3}$. The asymmetry is measurable to a level of a few percent. The decay rate asymmetry induced by the SSM may be well within reach of B factories.

The SSM has several new contributions to the EDMs of the neutron and the electron, yielding nontrivial constraints on the model parameters. In general, it is necessary that CP-violating phases are suppressed or supersymmetry particles are heavy. Nevertheless, as we have shown, there are sizable regions of parameter space in which the decay rate asymmetry of $B \rightarrow X_{s} \gamma$ becomes large. On the other hand, it may be possible [27 that the EDMs are suppressed by cancellation among different contributions while each contribution is not small. In this case, unsuppressed CP-violating phases with light supersymmetry particles could be allowed. In Fig. 7, discarding the constraints from the EDMs, we show parameter ranges for the $\mathrm{CP}$-violating phases $\alpha$ and $\theta$ in which the asymmetry is larger than 0.02 and those in which the branching ratio lies within the experimental bounds. The conditions are satisfied in dotted regions except the vicinities of CP-invariant points, i.e. $\sin \theta=\sin \alpha=0$, for the asymmetry. The $\mathrm{SU}(2)$ gaugino mass is taken

for $\widetilde{m}_{2}=200 \mathrm{GeV}$ and the $\tilde{t}_{1}$ mass is kept for $195 \mathrm{GeV} \leq \widetilde{M}_{t 1} \leq 205 \mathrm{GeV}$, with 
$\tan \beta=10$. The other parameters are set for the same values as in Fig. 3. We can see that the asymmetry is generally large for the parameter values consistent with the branching ratio, similarly to the results of our previous analyses. Maximal values of the asymmetry are of order 0.1. If the small values of the EDMs are due to the cancellation, the decay rate asymmetry is also likely to be large. It should be noted that the CP-invariant points are not allowed by the branching ratio in Fig. 7. For discussing the radiative $B$-meson decay in the SSM, neglect of $\mathrm{CP}$-violating phases could lead to incorrect conclusions.

The decay rate asymmetry for $B \rightarrow X_{s} \gamma$ in the SSM has also been studied recently under different assumptions. In Refs. [18, 28], assuming flexibility of masssquared matrices for squarks, the contributions by gluino-mediated and charginomediated diagrams are discussed and large values for the asymmetry are obtained. In the ordinary scheme based on supergravity and GUTs, however, the gluino contribution becomes much smaller than the chargino contribution, owing to a small mixing between $\tilde{b}_{L}$ and $\tilde{b}_{R}$. In Ref. 29], based on supergravity and GUTs, the chargino contribution is studied in a minimal model. Assuming universal values for soft-supersymmetry-braking parameters at the GUT scale, small values for the asymmetry are obtained, which is traced back to small CP-violating phases at the electroweak scale to satisfy the constraints from the EDMs [30]. However, if the universality or the minimality at the GUT scale is lifted, there is more freedom for the SSM parameters and their values adopted in our analyses could be well allowed.

We have studied the radiative $B$-meson decay, concentrating its decay rate asymmetry induced by a new source of CP violation in the SSM. The already available experiments give both directly and indirectly nontrivial constraints on the SSM parameters. In sizable regions of the parameter space allowed by these experiments, the asymmetry is predicted to have a magnitude larger than 0.01 . Such a magnitude of the asymmetry is larger than the SM prediction. Moreover, it may be possible to detect the asymmetry at B factories.

\section{Acknowledgments}

The authors thank M. Yamauchi for discussions on experiments at B factories. This work is supported in part by Grant-in-Aid for Scientific Research from the Ministry of Education, Science and Culture of Japan. 


\section{References}

[1] For reviews, see Y. Nir and H.R. Quinn, Annu. Rev. Nucl. Part. Sci. 42 (1992) 211 ;

M. Misiak, S. Pokorski, and J. Rosiek, Heavy Flavours II, p. 795 (World Scientific, Singapore, 1998);

J.L. Hewett, hep-ph/9803370 (1998).

[2] For reviews, see H.P. Nilles, Phys. Rep. 110 (1984) 1;

P. Nath, R. Arnowitt, and A.H. Chamseddine, Applied N=1 Supergravity (World Scientific, Singapore, 1984);

H.E. Haber and G.L. Kane, Phys. Rep. 117 (1985) 75.

[3] J.M. Soares, Nucl. Phys. B367 (1991) 575;

C. Greub, H. Simma, and D. Wyler, Nucl. Phys. B434 (1995) 39;

A. Ali, H. Asatrian, and C. Greub, Phys. Lett. B429 (1998) 87.

[4] N. Oshimo Nucl. Phys. B404 (1993) 20.

[5] G.C. Branco, G.C. Cho, Y. Kizukuri, and N. Oshimo, Phys. Lett. B337 (1994) 316; Nucl. Phys. B449 (1995) 483.

[6] M. Aoki and N. Oshimo, Nucl. Phys. B531 (1998) 49.

[7] M. Aoki, G.C. Cho, and N. Oshimo, hep-ph/9811251 (1998).

[8] M.S. Alam et al. (CLEO Collaboration), Phys. Rev. Lett. 74 (1995) 2885;

T. Skwarnicki (CLEO Collaboration), Talk given at the ICHEP98.

[9] R. Barate et al. (ALEPH Collaboration), Phys. Lett. B429 (1998) 169.

[10] A.L. Kagan and M. Neubert, Eur. Phys. J. C7 (1999) 5, and references therein.

[11] I.S. Altarev et al., JETP Lett. 44 (1986) 460;

K.F. Smith et al., Phys. Lett. B234 (1990) 191.

[12] S.A. Murthy et al., Phys. Rev. Lett. 63 (1989) 965;

D. Cho et al., Phys. Rev. Lett. 63 (1989) 2559;

K. Abdullah et al., Phys. Rev. Lett. 65 (1990) 2347;

E.D. Commins et al., Phys. Rev. A50 (1994) 2960. 
[13] X.-G. He, B.H.J. McKellar, and S. Pakvasa, Int. Jour. Mod. Phys. A4 (1989) 5011.

[14] W. Bernreuther and M. Suzuki, Rev. Mod. Phys. 63 (1991) 313.

[15] Y. Kizukuri and N. Oshimo, Phys. Rev. D45 (1992) 1806; Phys. Rev. D46 (1992) 3025.

[16] M. Aoki, A. Sugamoto, and N. Oshimo, Prog. Theor. Phys. 98 (1997) 1325;

S. Bar-Shalom, D. Atwood, and A. Soni, Phys. Rev. D57 (1998) 1495.

[17] A.J. Buras, M. Misiak, M. Münz, and S. Pokorski, Nucl. Phys. B424 (1994) 374.

[18] A.L. Kagan and M. Neubert, Phys. Rev. D58 (1998) 094012.

[19] N. Pott, Phys. Rev. D54 (1996) 938;

C. Greub, T. Hurth, and D. Wyler, Phys. Lett. B380 (1996) 385; Phys. Rev. D54 (1996) 3350.

[20] A. Ali and C. Greub, Phys. Lett. B361 (1995) 146.

[21] K. Chetyrkin, M. Misiak, and M. Münz, Phys. Lett. B400 (1997) 206, (E) B425 (1998) 414.

[22] K. Adel and Y.-P. Yao, Phys. Rev. D49 (1994) 4945;

C. Greub and T. Hurth, Phys. Rev. D56 (1997) 2934;

A.J. Buras, A. Kwiatkowski, and N. Pott, Nucl. Phys. B517 (1998) 353.

[23] M. Ciuchini, G. Degrassi, P. Gambino, and G.F. Giudice, Nucl. Phys. B534 (1998) 3.

[24] P. Ciafaloni, A. Romanino, and A. Strumia, Nucl. Phys. B524 (1998) 361;

M. Ciuchini, G. Degrassi, P. Gambino, and G.F. Giudice, Nucl. Phys. B527 (1998) 21;

F.M. Borzumati and C. Greub, Phys. Rev. D58 (1998) 074004.

[25] R. Barate et al. (ALEPH Collaboration), Phys. Lett. B434 (1998) 189;

K. Ackerstaff et al. (OPAL Collaboration), Eur. Phys. J. C6 (1999) 225.

[26] G. Abbiendi et al. (OPAL Collaboration), hep-ex/9809031 (1998);

P. Abreu et al. (DELPHI Collaboration), CERN-EP/98-176 (1998). 
[27] T. Ibrahim and P. Nath, Phys. Rev. D57 (1998) 478, (E) D58 (1998) 019901; Phys. Rev. D58 (1998) 111301;

M. Brhlik, G.J. Good, and G.L. Kane, hep-ph/9810457 (1998).

[28] C.-K. Chua, X.-G. He, and W.-S. Hou, hep-ph/9808431 (1998);

Y.G. Kim, P. Ko, and J.S. Lee, hep-ph/9810336 (1998);

S. Baek and P. Ko, hep-ph/9812229 (1998).

[29] T. Goto, Y.-Y. Keum, T. Nihei, Y. Okada, and Y. Shimizu, hep-ph/9812369 (1998).

[30] M. Matsumoto, J. Arafune, H. Tanaka, and K. Shiraishi, Phys. Rev. D46 (1992) 3966;

R. Garisto and J.D. Wells, Phys. Rev. D55 (1997) 1611. 


\begin{tabular}{ccccccccc}
\hline$i$ & 1 & 2 & 3 & 4 & 5 & 6 & 7 & 8 \\
\hline$a_{i}$ & $\frac{14}{23}$ & $\frac{16}{23}$ & $\frac{6}{23}$ & $-\frac{12}{23}$ & 0.4086 & -0.4230 & -0.8994 & 0.1456 \\
$h_{i}$ & $\frac{626126}{272277}$ & $-\frac{56281}{51730}$ & $-\frac{3}{7}$ & $-\frac{1}{14}$ & -0.6494 & -0.0380 & -0.0186 & -0.0057 \\
$\bar{h}_{i}$ & $\frac{313063}{363036}$ & 0 & 0 & 0 & -0.9135 & 0.0873 & -0.0571 & 0.0209 \\
\hline
\end{tabular}

Table 1: The values of $a_{i}, h_{i}$, and $\bar{h}_{i}$ in Eq. (3.9).
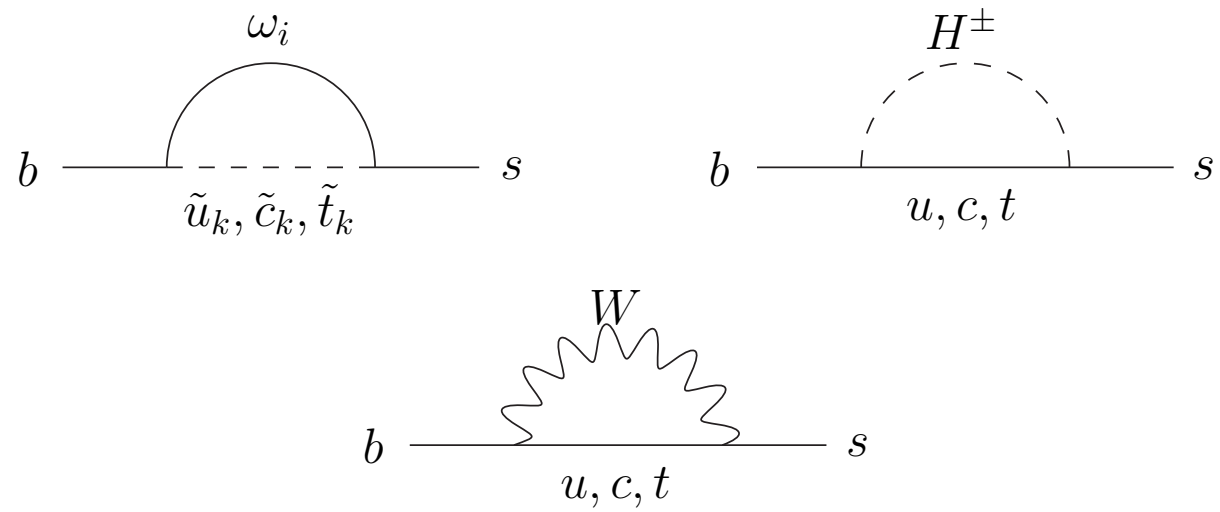

Figure 1: The one-loop diagrams which give contributions to $C_{7}$ and $C_{8}$ at the electroweak energy scale. The photon or gluon line should be attached appropriately. 


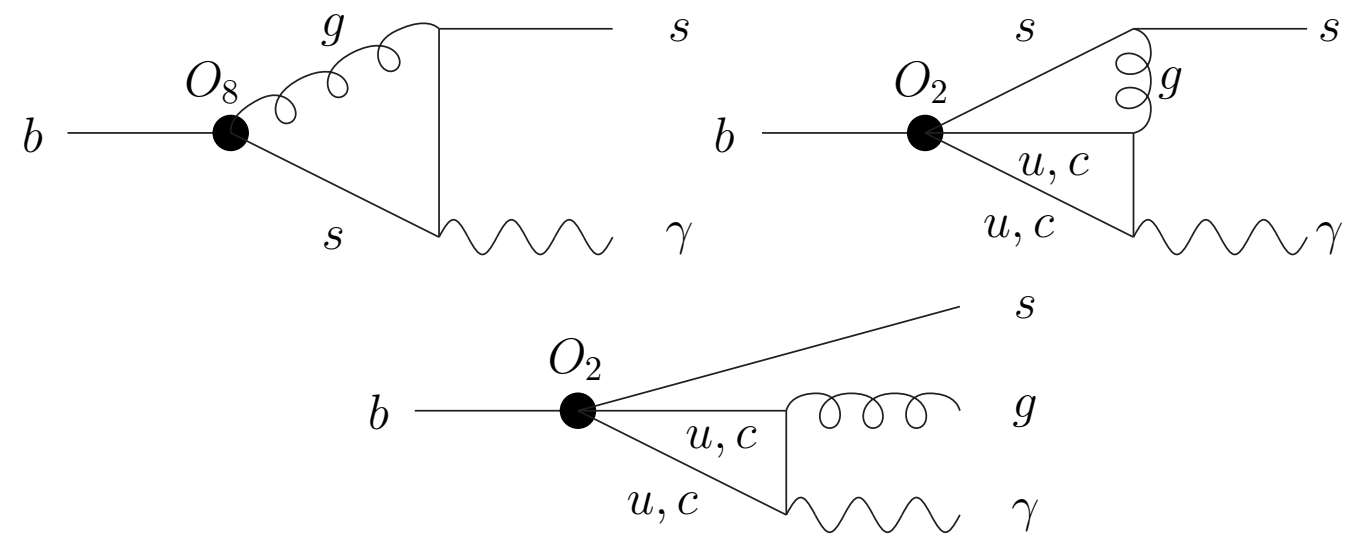

Figure 2: The loop diagrams with absorptive parts for $b \rightarrow s \gamma$ and $b \rightarrow s \gamma g$, where the blobs stand for the operators in Eq. (3.2). There are also similar diagrams with the photon line attached differently. 


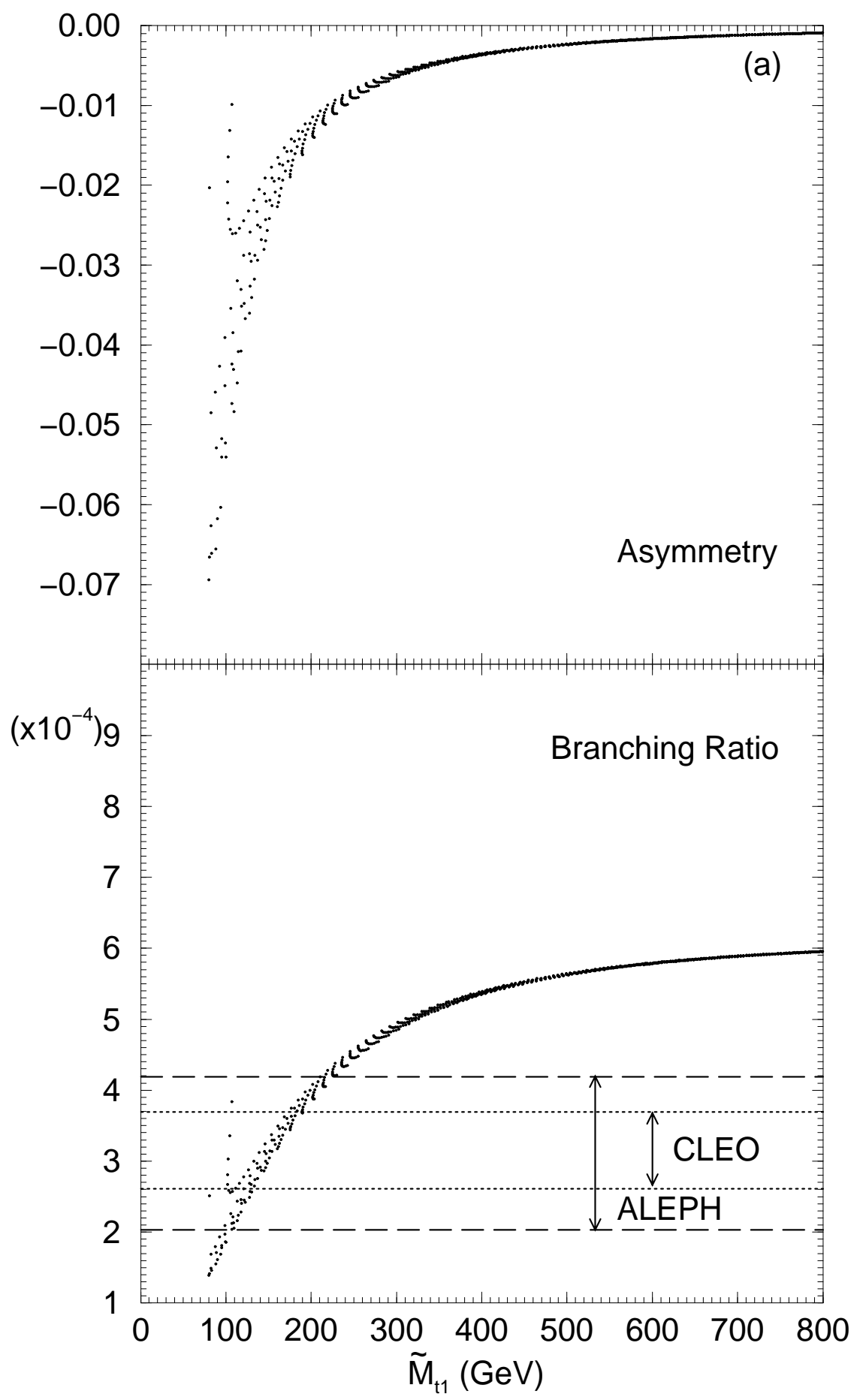




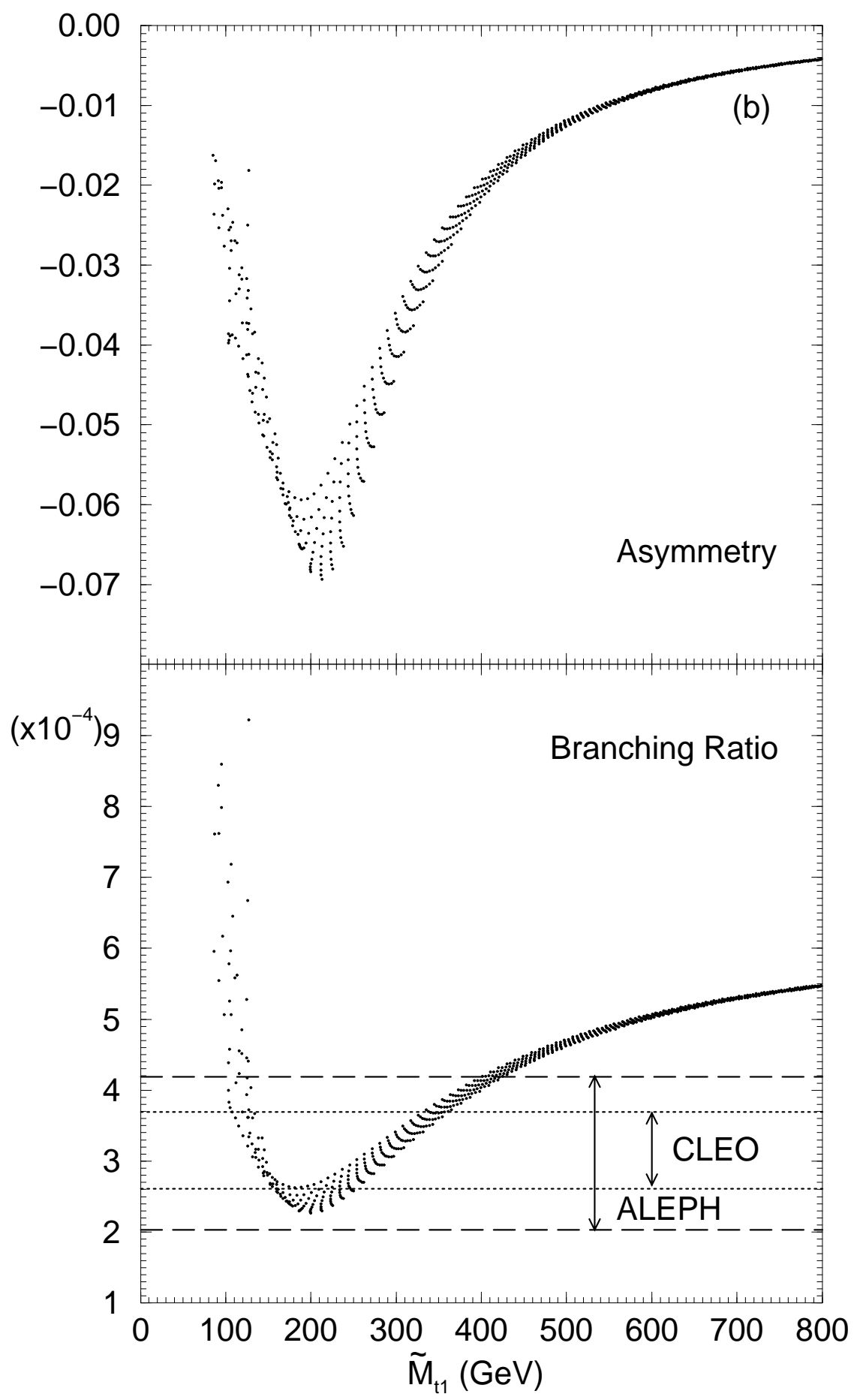




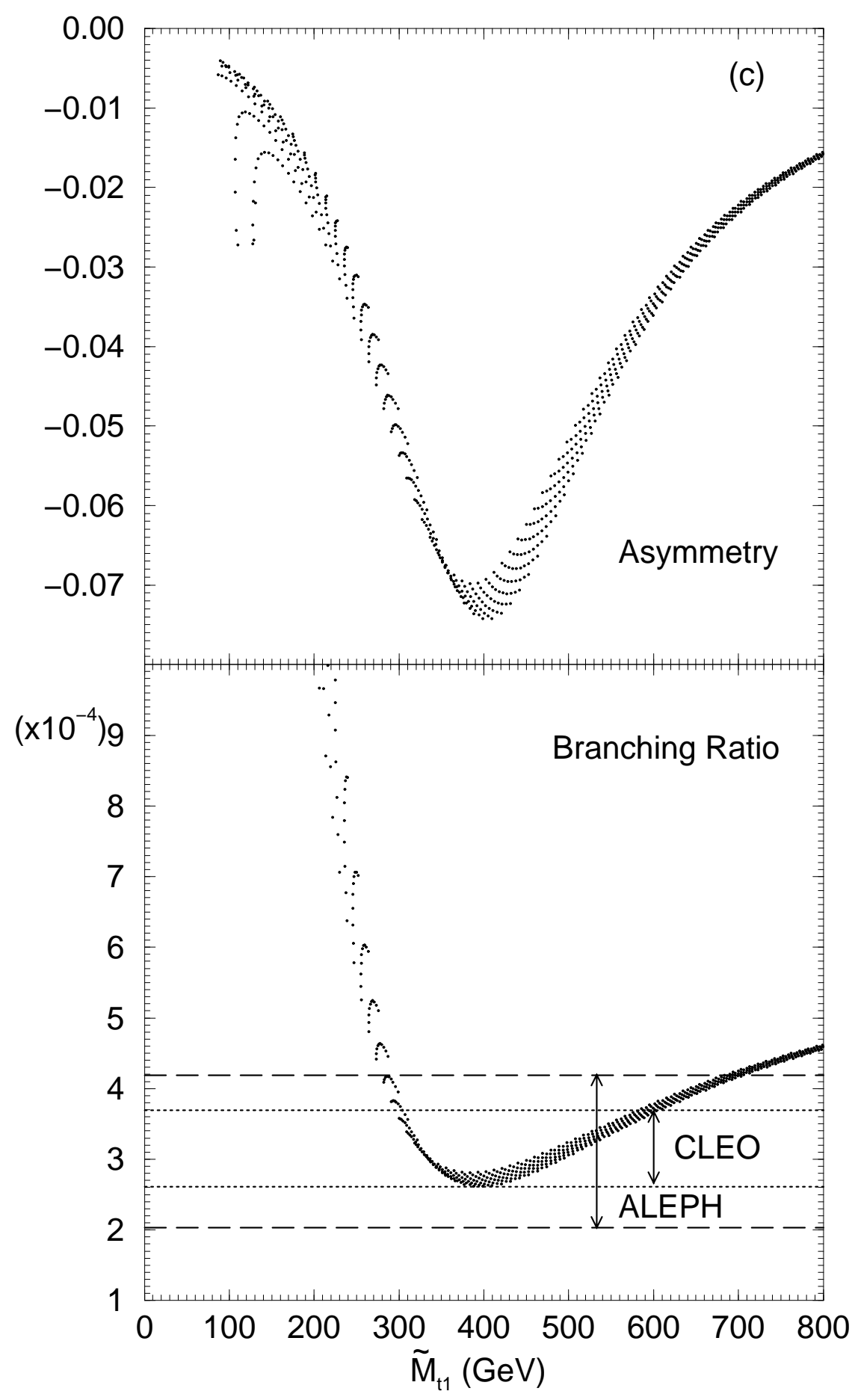

Figure 3: The decay rate asymmetry (upper) and the branching ratio (lower) of $B \rightarrow X_{s} \gamma$ for $R=1, \alpha=\pi / 4, m_{H}=100 \mathrm{GeV}$, and $M_{H^{ \pm}}=200 \mathrm{GeV}$. (a) $\tan \beta=2$, (b) $\tan \beta=10$, (c) $\tan \beta=30$. 


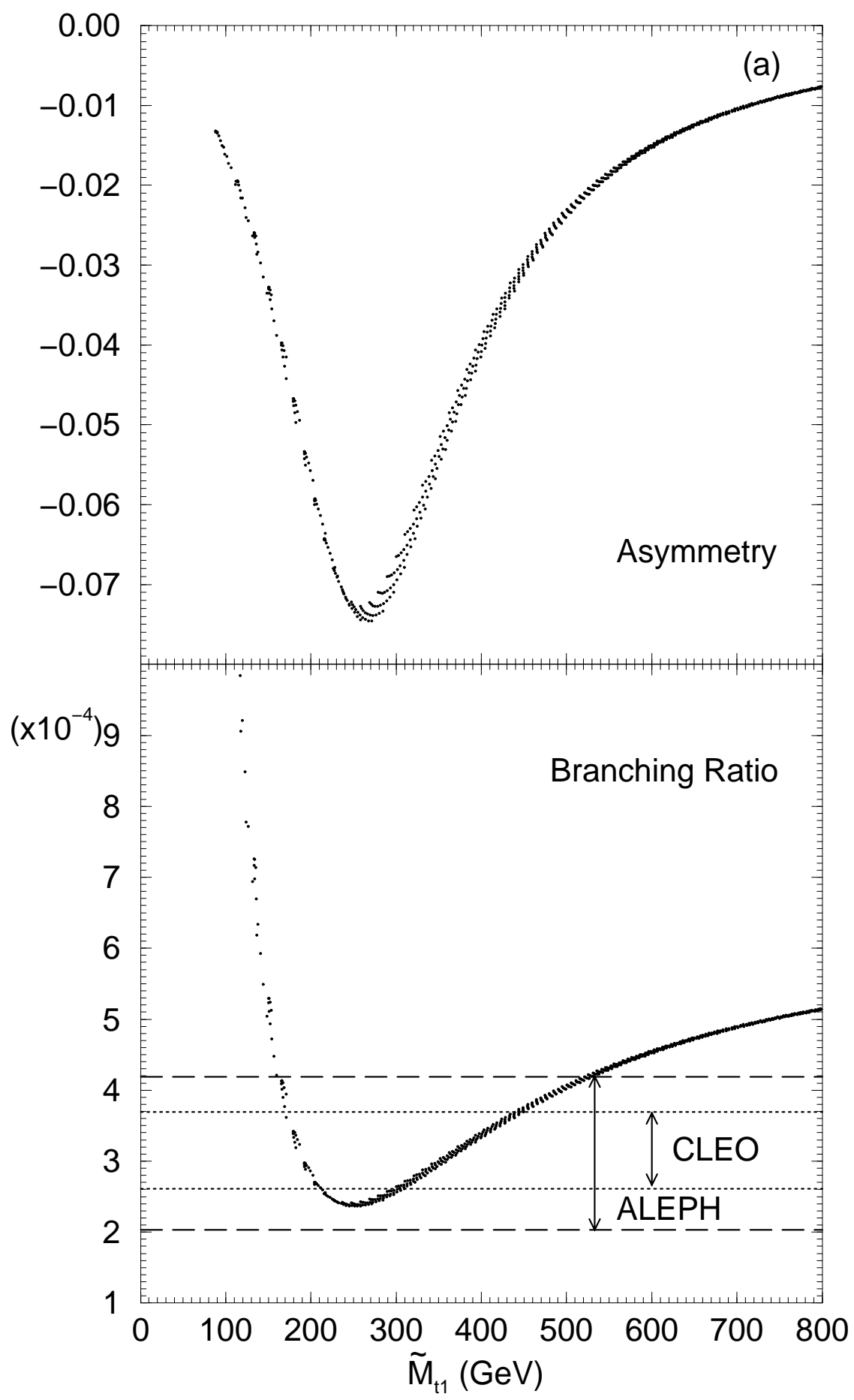




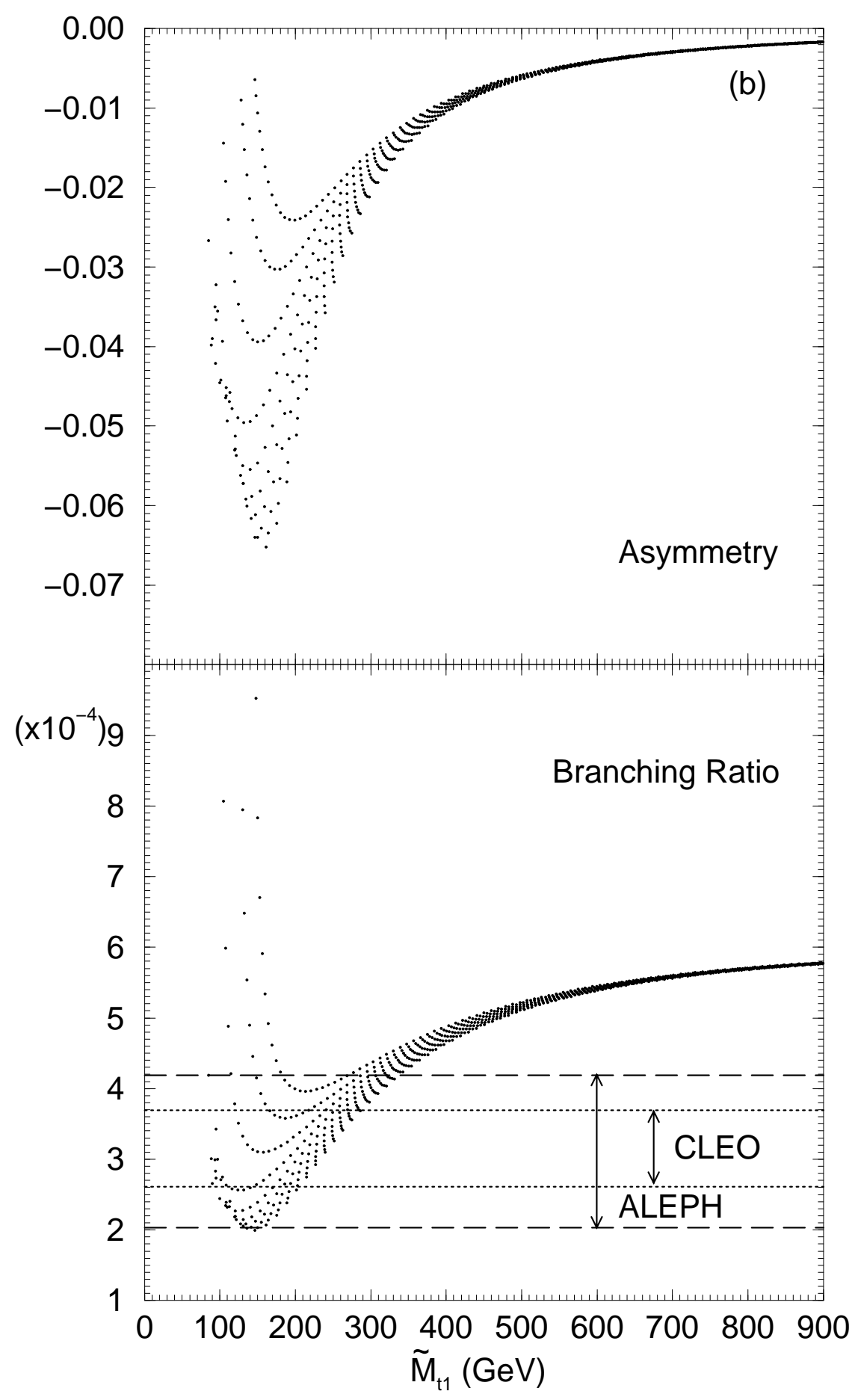

Figure 4: The decay rate asymmetry (upper) and the branching ratio (lower) of $B \rightarrow X_{s} \gamma$ for $\tan \beta=10, \alpha=\pi / 4, m_{H}=100 \mathrm{GeV}$, and $M_{H^{ \pm}}=200 \mathrm{GeV}$. (a) $R=0.5$, (b) $R=2$. 


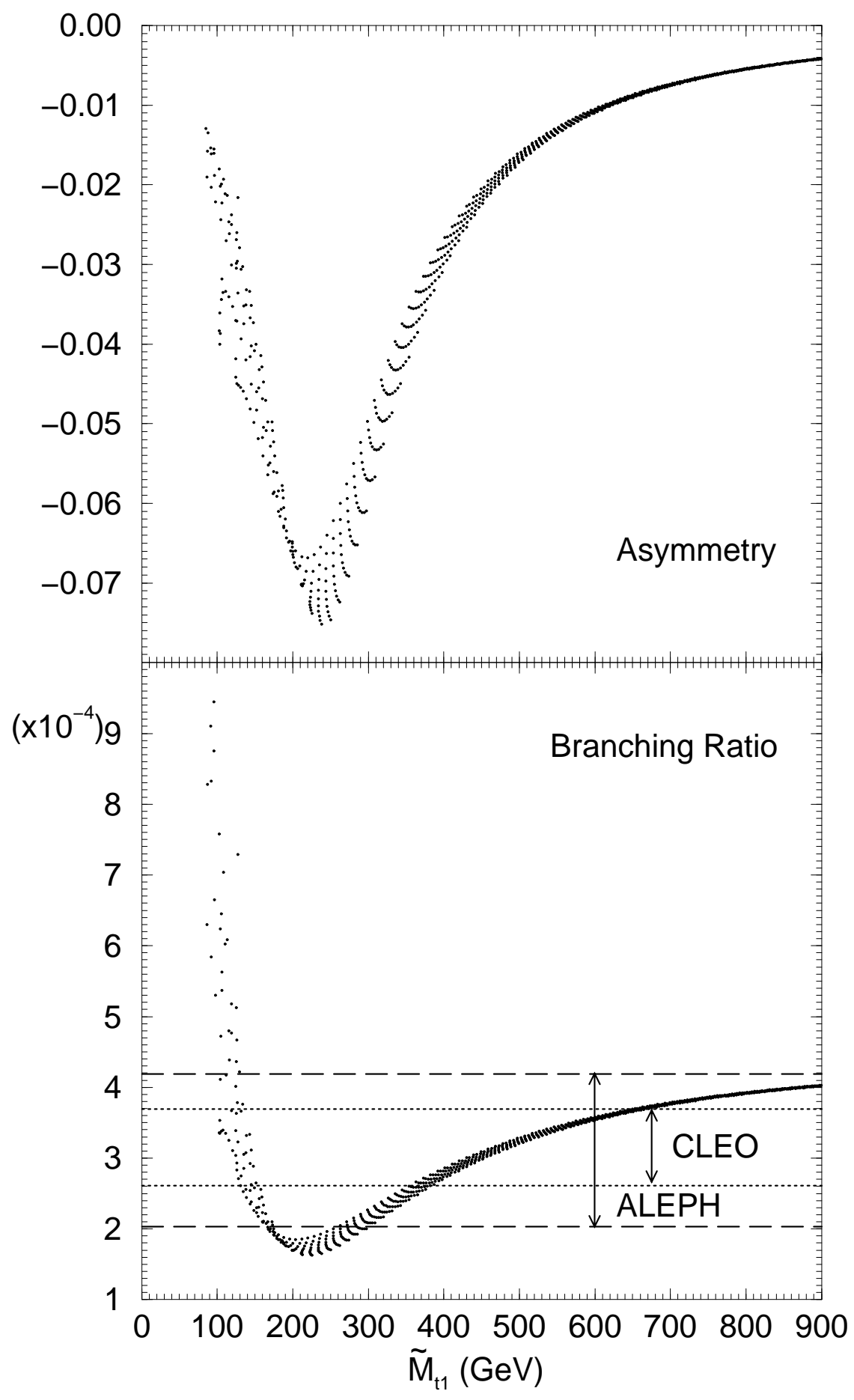

Figure 5: The decay rate asymmetry (upper) and the branching ratio (lower) of $B \rightarrow X_{s} \gamma$ for $M_{H^{ \pm}}=500 \mathrm{GeV}, R=1, \tan \beta=10, \alpha=\pi / 4$, and $m_{H}=100 \mathrm{GeV}$. 


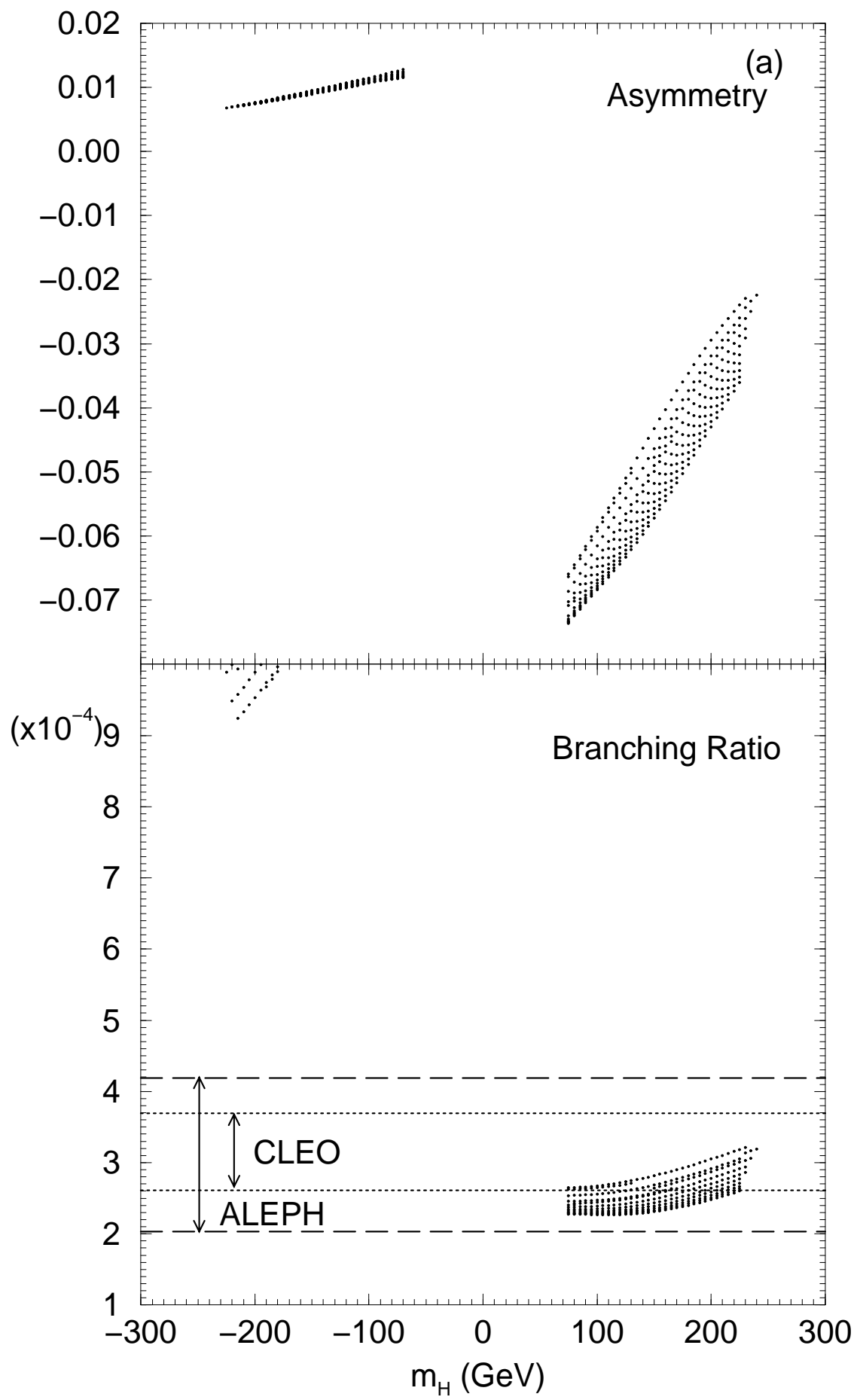




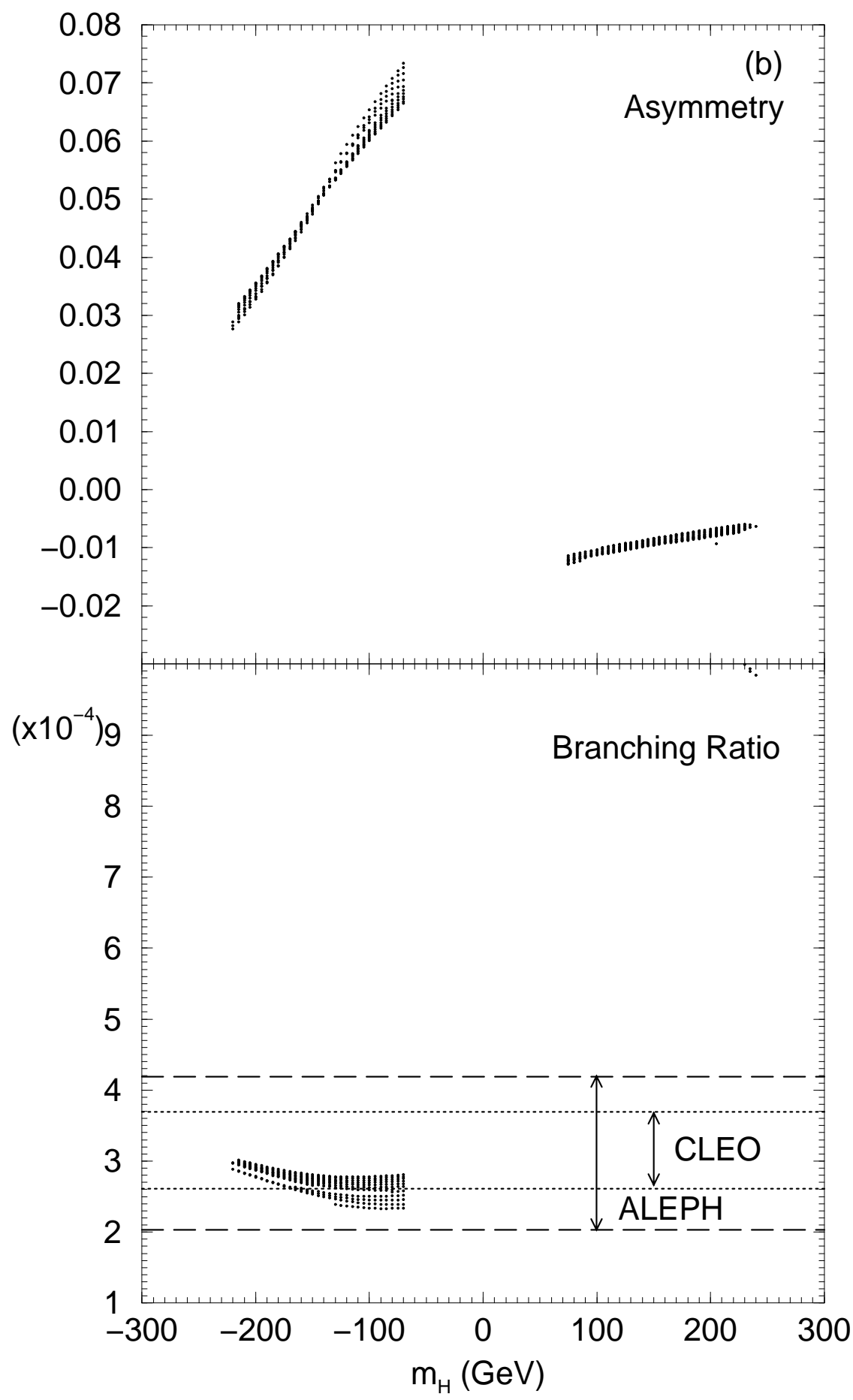

Figure 6: The decay rate asymmetry (upper) and the branching ratio (lower) of $B \rightarrow X_{s} \gamma$ as functions of $m_{H}$ for $R=1, \tan \beta=10$, and $M_{H^{ \pm}}=200 \mathrm{GeV}$. The mass of $\tilde{t}_{1}$ is taken for $195 \mathrm{GeV} \leq \widetilde{M}_{t 1} \leq 205 \mathrm{GeV}$. (a) $\alpha=\pi / 4$, (b) $\alpha=3 \pi / 4$. 


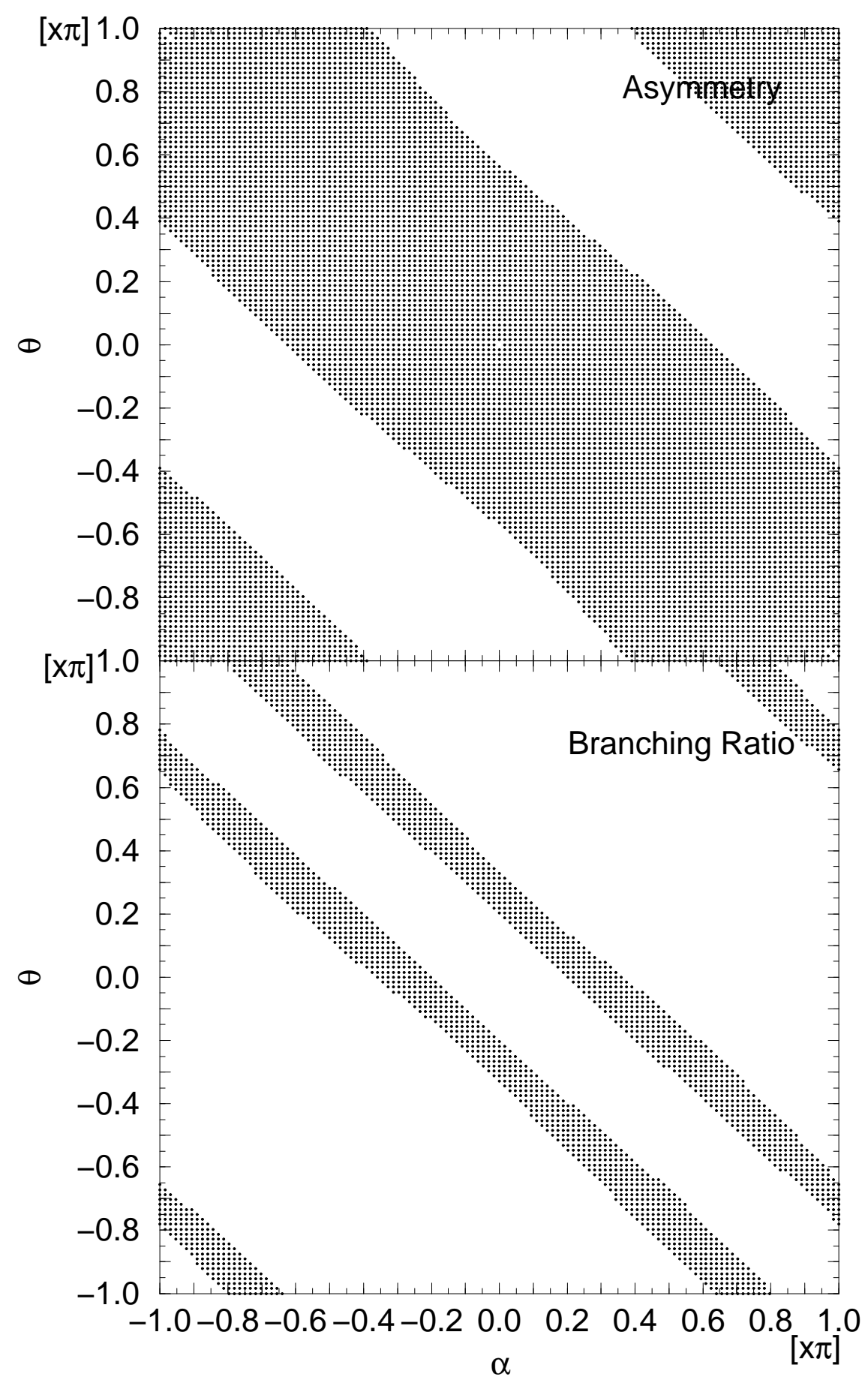

Figure 7: The regions (dotted) in the $(\alpha, \theta)$ plane for $\left|\mathcal{A}_{C P}\right| \geq 0.02$ (upper) and for the branching ratio allowed by the ALEPH experiment (lower). The parameter values are set for $\widetilde{m}_{2}=200 \mathrm{GeV},\left|m_{H}\right|=100 \mathrm{GeV}, 195 \mathrm{GeV} \leq \widetilde{M}_{t 1} \leq 205 \mathrm{GeV}$, $R=1, \tan \beta=10$, and $M_{H^{ \pm}}=200 \mathrm{GeV}$. 